\title{
A Syndrome of Acute Cerebellar and Hippocampal Restricted Diffusion: A Potentially Fatal Stroke Mimic
}

\author{
Adam Jasne MD, ${ }^{1}$ Matthew Smith MD, ${ }^{1}$ Khalid H Alsherbini MD, ${ }^{2}$ Abhi Pandhi MD, ${ }^{2}$ Nitin \\ Goyal MD, ${ }^{2}$ Daniel Kanter MD1 \\ 1: University of Cincinnati, Department of Neurology and Rehabilitation Medicine; \\ 2: University of Tennessee Health Science Center, Neurology Department
}

\section{Introduction}

Abnormally restricted diffusion on $\mathrm{MRI}$ is often associated with ischemic stroke, but other conditions can present similarly. We present six cases of an unusual pattern of restricted diffusion in the bilateral hippocampi and cerebellar cortices leading to obstructive hydrocephalus.

Patients present with decreased level of consciousness and require osmotic therapy and/or CSF flow diversion. This pattern of injury is distinct from findings typical for ischemic, anoxic, or toxic injury, suggesting it may represent an under-recognized syndrome which we term CHANTER (Cerebellar, Hippocampal, And basal Nuclei Transient Edema with Restricted diffusion).

\section{Methods}

- We identified an ad hoc series of patients at our two institutions between years 2014 and 2017 who presented to the neurosurgical ICU with severe, otherwise-unexplained cerebellar edema.

- We retrospectively identified several commonalities in history, presentation, and imaging.
Figure. Diffusion-weighted MRI of selected brain regions

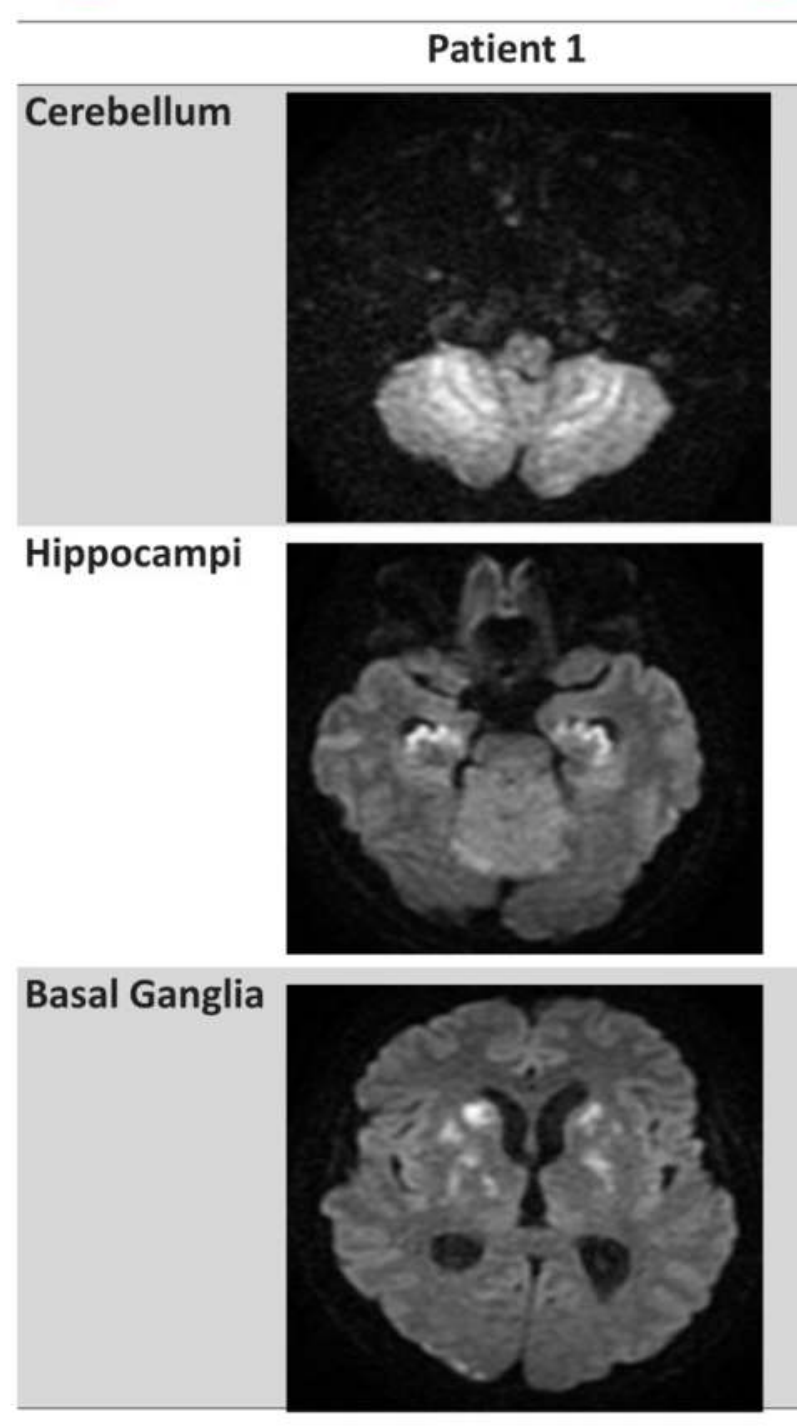

Restricted diffusion shown here was correspondingly hypointense on apparent diffusion coefficient mapping (not shown). Cases from University of Tennessee Health Science Center (not shown)

demonstrated a similar pattern.
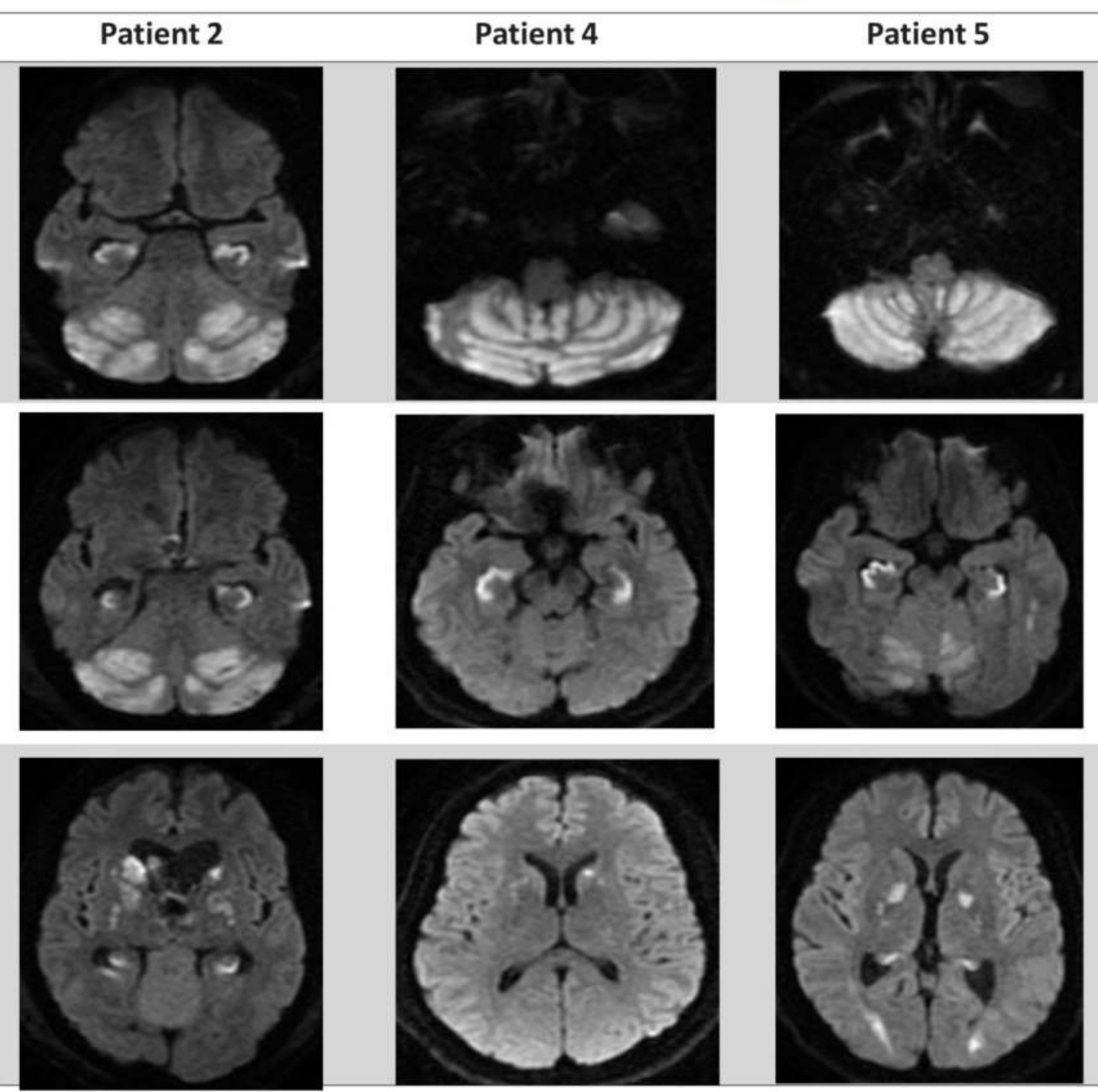

\section{Results}

Between two institutions, we identified six patients with similar presentations of decreased responsiveness and hypoxia in the context of exposure to opiates or other exposures (table). Ages ranged from 33 to 59 years, and four patients were male. All patients demonstrated similar imaging findings (figure) and all required medical or surgical intervention due to obstructive hydrocephalus secondary to cerebellar edema. The five patients who survived to discharge demonstrated variable degrees of physical and memory impairment on discharge and follow up.

Table. Patient characteristics and outcomes

\begin{tabular}{|c|c|c|c|c|c|c|}
\hline & Patient 1: 54F & Patient 2: 59M & Patient 3: 49F & Patient 4: 50M & Patient 5: 35M & Patient 6: 33N \\
\hline $\begin{array}{l}\text { Acute } \\
\text { exposure }\end{array}$ & hydromorphone & $\begin{array}{l}\text { BZD, cocaine, } \\
\text { opiates }\end{array}$ & heroin & $\begin{array}{l}\text { cocaine, } \\
\text { fentanyl }\end{array}$ & $\begin{array}{l}\text { amphetamine, } \\
\text { etOH }\end{array}$ & $\begin{array}{l}\text { cocaine, BZD, } \\
\text { etOH }\end{array}$ \\
\hline $\begin{array}{l}\text { Clinical } \\
\text { presentation }\end{array}$ & $\begin{array}{l}\text { unresponsive, } \\
\text { apneic after } \\
\text { in-hospital } \\
\text { hydromorphone }\end{array}$ & $\begin{array}{l}\text { unresponsive, } \\
\text { extensor } \\
\text { posturing, } \\
\text { hypoxic }\end{array}$ & $\begin{array}{l}\text { found } \\
\text { unresponsive, } \\
\text { cyanotic }\end{array}$ & $\begin{array}{l}\text { found } \\
\text { unresponsive; } \\
\text { last well } 2 \text { days } \\
\text { prior }\end{array}$ & $\begin{array}{l}\text { encephalopathy, } \\
\text { decreased } \\
\text { activity }\end{array}$ & $\begin{array}{l}\text { found } \\
\text { unresponsive }\end{array}$ \\
\hline Initial GCS & 3 & 5 & 7 & 3 & 11 & 3 \\
\hline $\begin{array}{l}\text { Hospital } \\
\text { interventions }\end{array}$ & $\begin{array}{l}\text { MV, HTS, EVD, } \\
\text { SubOcc }\end{array}$ & $\begin{array}{l}\text { MV, HTS, EVD, } \\
\text { SubOcc }\end{array}$ & $\begin{array}{l}\text { MV, HTS, EVD, } \\
\text { SubOcc }\end{array}$ & $\begin{array}{l}\text { MV, HTS, EVD; } \\
\text { had pre- } \\
\text { existing } \\
\text { SubOcc }\end{array}$ & HTS & MV, HTS \\
\hline Disposition & $\begin{array}{l}\text { trach/PEG, } \\
\text { acute } \\
\text { rehabilitation }\end{array}$ & $\begin{array}{l}\text { in-hospital } \\
\text { death }\end{array}$ & $\begin{array}{l}\text { PEG, shelter } \\
\text { home }\end{array}$ & SNF & $\begin{array}{l}\text { acute } \\
\text { rehabilitation }\end{array}$ & $\begin{array}{l}\text { home with } \\
\text { supervision }\end{array}$ \\
\hline Followup & $\begin{array}{l}2 \text { months: } \\
\text { moderate } \\
\text { cognitive } \\
\text { impairment; } \\
\text { mild-moderate } \\
\text { weakness, } \\
\text { wheelchair- } \\
\text { bound }\end{array}$ & n/a & $\begin{array}{l}1 \text { month: mild } \\
\text { dysarthria only }\end{array}$ & $\begin{array}{l}6 \text { months: } \\
\text { cognitive and } \\
\text { memory } \\
\text { deficits; living } \\
\text { at nursing } \\
\text { home }\end{array}$ & $\begin{array}{l}3 \text { months: } \\
\text { abulia, memory } \\
\text { and cognitive } \\
\text { issues, mild } \\
\text { hemiparesis; } \\
\text { living with family }\end{array}$ & $\begin{array}{l}2 \text { years: } \\
\text { end-stage } \\
\text { renal disease; } \\
\text { living alone } \\
\text { with home } \\
\text { health }\end{array}$ \\
\hline
\end{tabular}

\section{Conclusions}

- CHANTER is potentially novel syndrome of cerebellar, hippocampal, and basal nuclei restricted diffusion

- CHANTER is distinct from anoxic brain injury, ischemic stroke, and toxidromes such as "chasing the dragon."

- Aggressive early management of cerebellar edema and hydrocephalus may be warranted, given the potential for good outcomes 